\title{
Identification of mobile vehicle through multilayer intercommunication
}

\author{
Ranjan Goyal, Anuradha Gnanaprakasam, Paritosh Pal Singh \\ School of Computer Science and Engineering, Vellore Institute of Technology, Vellore, India
}

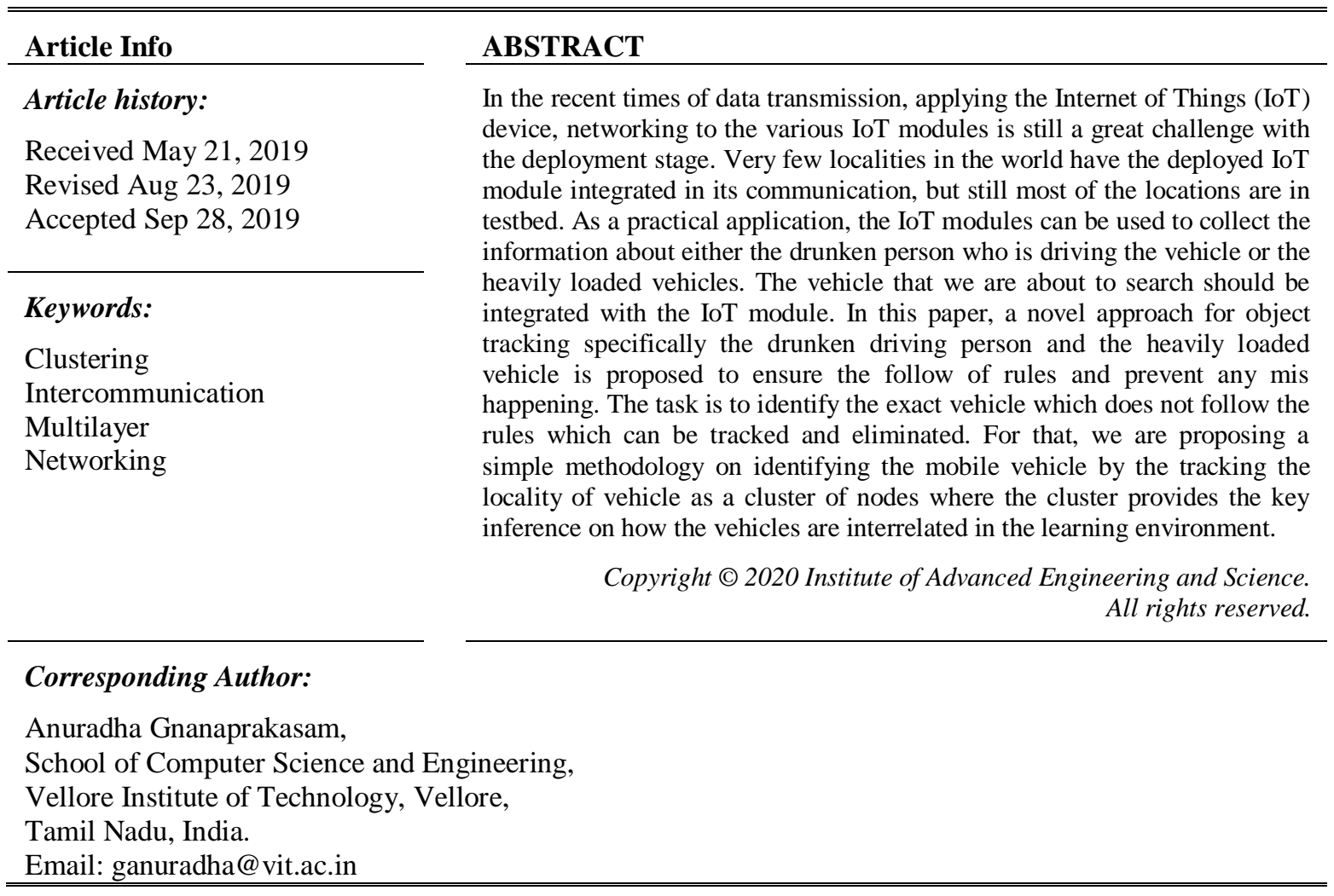

\section{INTRODUCTION}

Due to increasing road accidents and mis happenings, it has become really important to keep a check on the vehicles and ensure the follow of rules on the road. The main focus is required on the activities including drunken person driving the vehicle and heavily loaded vehicles. In India, everyday many people lose their life by becoming a victim of the mis happening caused by these activities. Thus, in order to prevent these accidents, it is highly required to implement a mechanism that can ensure follow of rules on road by everyone. In this paper, a novel approach is proposed that helps in object tracking for these activities with the help of cellular networks. The identification of the vehicle zone is the most important task in order to find the object and track it down if required. For this purpose, the communication between the nodes i.e. the cellular device is required to be established. Once the connection is established, the vehicle data can be obtained with the help of Internet of Things (IoT) modules integration. Here, for the integration of various IoT modules with the help of networking concepts, there is no option of explaining about the IoT. The work is entirely focused on integration or intercommunication between the various IoT modules.

Here, in terms of vehicle driving abnormality reasons are more specifically focused towards the drunken person driving and heavily loaded vehicles. The same is applicable on similar situations of abnormality in vehicle driving on the road. The work in reference [1] provided a solution for mobile phonebased detection for drunk driving with the utilization of accelerometer and orientation sensor. Nevertheless, the work focused only on detection of such scenario. In such case, the intimidation to the road safety officers can be made in order to ensure the elimination of such vehicle that may later lead to any mis happening. In the reference [2], the work provided a road anomalies detection system using Vehicle to Vehicle (V2V) 
network and cluster features with the utilization of cars distance to warn the nearby vehicles of incoming dangers including road block or any accidents. The work considered a modified k-means algorithm in order to count the dynamic clusters based on number of nodes. Here, on formation of clusters, the vehicles cooperate to determine the condition of the area which is used for determination of the anomalies. This information is then broadcasted to nearby clusters in order to warn about the potential danger. Nevertheless, the task of object tracking is still required to be established and this work is focused on the same. The tasks required in order to achieve the same is discussed below.

\subsection{Tasks Required to be Performed}

The first task is to establish the communication between the nodes. For this purpose, the identification of the nodes in the zone is required zone in which the vehicle is present. This is performed with the utilization of clustering on cellular networks. Here, the clustering is an important part in the task of choosing the head node. The clusters are formed based on the given limits or some parameters for the given network of nodes. The election algorithm is required to select the cluster head local clusters. So, Initially, the identification of random nodes is performed depending on the area under which the data collection is about to be performed. Once the node is defined, the nearest neighbors of that node are identified with the help of various distance factors such as Euclidean distance, Manhattan distance and Mahala Nobis distance. Here, the work considers the Mahala Nobis distance due to dynamic mobility of the nodes. Also, the energy factor is considered because of the network nodes which are assumed with large energy. Here, the energy gets drained based on the transmission of the data. As the device battery cannot hold for longer duration of time, so to maintain the energy, the work focuses on cluster head determination. When the energy of the node goes below the threshold level or neighbor's energy, the cluster head is determined based on the election algorithm. Also, this whole scenario will be timestamp based i.e. the cluster formed will be allotted certain time limit in which it can perform the data transmission. A timestamp based existing algorithm is discussed in the Section 2.

The next task in this work is to find out one cluster head in each of the formed clusters. For this, the election for cluster selection is performed using the election algorithm. The cluster head is selected using the election algorithm that considers the given energy levels of the nodes. These energy levels are used to set the priorities of nodes present in the cluster. Now during the election, the energy (E) and distance (d) of each node is considered in that cluster that sets the priorities of each node in the cluster. From this, we get the head of that cluster. Now, this cluster head can be used to transmit data to any node within its maximum transmission range. Also, the work considers the dynamic nature of the nodes. In case of addition of any new node or in case of failure of any existing head node in the preformed cluster, election is done again to find the new cluster head.

\section{EXISTING MECHANISMS}

\subsection{Clustering Algorithm}

Clustering is an important requirement in this work. The clustering algorithm required in this research is distance based dynamic clustering [3]. The k-means clustering is a method of clustering based on the centroid and distances of the nodes present near to each other. The $k$-means clustering partitions the $n$ number of nodes into $\mathrm{k}$ clusters based on the centroid and distance. The centroid gets shifted after every iteration and distance is calculated again. This process is repeated till the clusters formed becomes same after the next iteration.

\subsection{Data Preservation and Head Election}

To perform the required tasks for this work discussed in the Section 1, the existing algorithms used in the proposed methodology are given as follows:

a) Ricart-Agarwala Algorithm: The Ricart-Agarwala is a basic improvement over Lamport timestamp algorithm. There are three major factors in this algorithm: Node (Ni), Timestamp (TS) and Message (m). The node sends a message with a timestamp. The lesser timestamp value means greater priority for the node to be chosen. After certain time, when there are different timestamp and messages for different nodes, only one node can enter into the critical section. So, the node with least timestamp is taken.

b) Suzuki Kazama Algorithm: The Suzuki Kazama Algorithm is a token-based algorithm. In this algorithm, three queues are maintained: Request queue, Last queue and CS queue. In request queue, if $\mathrm{Ni}$ wants to request for token then update the $\mathrm{Ni}$ in request queue and send it to every other node. The Last queue tells from where request queue has come from recently. The CS queue keeps the processes that request for entering into the critical section. 
c) Bully Algorithm: The Bully Algorithm is an election algorithm that determines which node will play the role of coordinator or server in the given network of nodes. The algorithm works on the agreement of all the nodes for starting the election and any node can start the election at any time. The bully algorithm can handle the crash failures but it cannot handle the communication related failures. According to this algorithm, if the coordinator fails at any point of time, any other node can conduct the election for selection of the new head or coordinator. This algorithm is known for guarantying safety and liveness and due to this property, they are more suitable for real life scenarios.

\section{RELATED WORKS}

There are several works proposed by researchers in the field of networking related to the work done in this paper. Paul et al. [4] proposed a cluster-based leader election algorithm for mobile ad hoc networks. The work proposed a method for clustering the network by avoiding the dynamic nature of the sub network up to a particular threshold during the leader election procedure. The work considered the strategy to avoid power consumption and considered the re-election scenario only in the case of failure of the leader node. The work also defines the concept of local and global leader based on the formed cluster.

Ghai et al. [5] proposed a method for energy efficient dynamic nearest node election based on RSSI and cooperative communication between the nodes. The algorithm finds the localization of the node and tries to improve the time and energy efficiency. Kumar et al. [6] proposed a work based on dynamic weight adjustments by soft computing that has non-deterministic algorithm like fuzzy logic and neural networks. The work was focused on selecting the cluster head based on the appropriate weights for dynamic nodes with less computation overhead.

Alabass et al. [7] proposed a dynamic cluster head election mechanism that calculates the residual energy value to determine the candidacy of each node in the cluster to become the cluster head. In this mechanism, each sensor node compares their residual energy level to the other nodes in a cluster and based on this residual energy level the cluster head is selected [8]. Mehr [9] proposed an algorithm for cluster head election that is based on the imperialist competitive algorithm. The work tries to overcome the energy consumption in clustering that can help in improving the network lifetime. The proposed algorithm by Mehr [9] was compared with the LEACH protocol [10-11] using the MATLAB simulation.

The issue in the model proposed by Paul et al. [4] is that the work did not consider the dynamic nature of the nodes, so in case of any new entry of a node, the cluster is not modified and hence no modification in the cluster head selected. The rest of the literature also fails to focus on some of the important facts and parameters like proper data preservation and distances during election and clustering respectively. Also, the challenges of Machine to Machine (M2M) massive access in Wireless Cellular Networks (WCN) are required to be considered in order to achieve the objective of this paper. The same is discussed below.

\subsection{Considerations and Challenges in WCN}

In the cellular systems, the major requirement that concerns Human to Human $(\mathrm{H} 2 \mathrm{H})$ services are higher data rates, lower power consumption and better support for mobility of users. Also, the bandwidth is limited. This can be due to the point that GSM network faces the serious capacity issue in the presence of the synchronized access of a large number of Machine to Device (M2Ds). The improvements in the core network are being more focused and at the higher layers of the radio network, considering the main aspect i.e. identification of the M2Ds within a network considering the short messaging service and the load balancing. But, the practical and technical limitations prevent Global System for Mobile (GSM) to continue the service in the long run. These include the point that the number of M2Ds that can be connected to single GSM Base Station (BS) is limited. Also, in the LTE cellular service, when it comes to support the M2M in the LTE model, there are a number of challenges including control overhead, coverage extension, energy efficiency, security issue, robustness and scalability. So, in offering the LTE standard during the large number of M2Ds will result in conventional service quality degradation due to long access delays and high access failure rate. The M2M services will also be affected by these impairments but it will be less significant as compared to the significance for the conventional services. Still, the overload due to computation work and energy consumption will be critical for M2D applications [12].

Now, one way to handle the massive access to the base station is by electing the cluster heads for the remaining terminal nodes. By doing this, the number of access requests to the base station will get limited to the cluster heads. Also, proper election of the cluster head can help in decreasing the energy consumption of the system, by performing multi hop transmissions over the high gain links instead of direct transmissions over poor quality links. Consider one solution which provides energy efficient clustering of M2Ds. In this approach, the number of simultaneous accesses to the base station and the energy consumption of M2Ds is limited. Now, to minimize the energy consumption while keeping the number of clusters below a certain 
threshold, the number of access requests to the base station will be limited and redundant signaling of $\mathrm{M} 2 \mathrm{M}$ devices will be reduced.

The authors [13] proposed some combinations of clustering and cluster head election algorithm. By using the $\mathrm{K}$ means algorithm for grouping and then selecting the cluster heads randomly in the M2Ds. Then the procedure of clustering is iterated until the clusters remains same for the next iteration. In the case of K Maximal Channel Gain, the cluster heads are simply selected based on the M2Ds with highest channel gain towards the base station and the Optimal Energy Consumption implements an exhaustive search for the head that minimizes the energy consumption within each of the clusters returned by the $\mathrm{K}$ means algorithm. The simulation of the techniques has shown that these techniques are helpful in reducing the massive access issue and improving the energy efficiency. Though all algorithms performed better than direct transmission between the M2Ds and base station in terms of both channel contention and consumption of energy, for all clustering algorithms, cluster heads are subjected to high power consumption and therefore they may fail because of the energy depletion before the other nodes. Thus, the mechanism of the dynamic clustering should be considered along with the suitable cluster head election algorithm.

Based on the above considerations and issues, the dynamic clustering is chosen instead of the static one as the devices are considered to be mobile i.e. the devices are moving. So, in this scenario, the static clustering will fail as the device will get out of the range. Also, the communication between the devices is required to be established so as to discover the nodes. The work also suggests to send the data to the other end so as to get the information of the nodes which can be further used for performance analysis. It is also a notable point that in the attempt to reduce the power uplink, the transmit bandwidth and the complexity of the baseband components may result a decrease in the cost and the power consumption of the chain but this may result in lower coverage range. In $5 \mathrm{G}$ cellular network, massive multiple input multiple output can result in enlarged number of simultaneous transmissions without burdening the nodes. This may help in supporting massive M2M access. Now, to deal with the increasing number of devices in hot spot areas, the small cells can be employed for the densification of the network.

Thus, based on the literature and related works [14-21], a methodology is proposed in this paper that considers all the issues and challenges faced in the wireless cellular networks and deals with the same by utilizing the dynamic clustering and a coordinator selection algorithm. The same is discussed in the next section.

\section{PROPOSED METHODOLOGY}

The work proposed in this paper is based on the idea of the existing mechanisms discussed in the Section 2. The idea is to cluster the given networking nodes in the infrastructures. The infrastructures or beacons are the hexagonal shaped areas in a network that contains the networking devices which are also called as nodes. These nodes have different frequencies based on the type of the device like Bluetooth or WiFi device or any other networking device. These devices or nodes can communicate with each other within their range of frequency. The nodes can move from one position to the other in the network. The infrastructure or cell can have some number of nodes that have their own radial beacons i.e. frequency range of transmission.

Thus, this paper proposes a methodology to form a cluster in a given network of nodes considering the energy and distance parameters. The clustering method used in this approach is based on the idea of clustering that considers the distance and energy of the nodes forming the cluster. The distance taken into consideration for forming the cluster is based on radial beacons i.e. the frequency range of transmission of the nodes. Thus, the clusters can be formed using this methodology. Figure 1 shows the procedural flowchart for the proposed clustering mechanism.

Initially, the nodes present in the network are identified and some node (n) is selected. Now, the next step is to check if some other node $(x)$ present in the proximity range of $n(P(n))$. If some $x$ is present, then the energy level of the nodes is checked. Then, if the energy of the current node (Ec) is found to be greater than the energy of the previous node (Ep), i.e. Ec > Ep then that current node is updated into a cluster formation. After this, time $t(c)$ is allotted to the formed cluster. In the middle of the procedure, if the $x$ is not found in the $\mathrm{P}(\mathrm{n})$ then the flow checks for any new entry of node. In case of any new entry, the same procedure is followed starting from the $\mathrm{x}$ in $\mathrm{P}(\mathrm{n})$ check. Also, in case of the Ec $>$ Ep check if the condition fails, then the flow checks for any new entry of node which once again checks for the proximity of the remaining nodes with the repetition of the entire procedure. Further, in case the new entry is not found or in case the time expiration condition fails, the flow moves to the procedure starting from checking the energy level of the node. Figure 2 depicts the cluster formation in an arbitrary network of nodes using the proposed clustering mechanism. 
Now, after the formation of the clusters, the cluster head is required to be selected. For the selection of the cluster head, the election algorithm comes into the scenario. Here, the election is performed using the bully algorithm. The bully algorithm will consider the distance and energy parameters and will conduct election to select the cluster head. Figure 3 shows the procedural flowchart for the proposed cluster head selection mechanism.

Here, initially the flow identifies the cluster head in a cluster and checks if the cluster head is active or not. If the cluster head is active then the flow waits for some time and checks again. In case the cluster head is not active then cluster election procedure is started. Initially, the max energy (Emax) is initialized to zero. Now, the flow waits for time $t^{\prime}$ and performs data preservation. Now the clusters are initialized and a cluster is selected. Now, for that cluster, get energy of all nodes. Now, for some initial node, check if the energy of that node (Ec) is greater than Emax i.e. Ep > Emax condition is satisfied or not. If the condition is satisfied, then that node is selected as cluster head. In case the condition is not satisfied, then the flow is sent to identify the unselected nodes. Now, from the cluster head selection, the flow is sent to check for any unselected nodes. In case of presence of any unselected nodes, the flow is sent to identify the unselected nodes and in case of no unselected nodes the flow is sent to check for any unselected clusters. If the unselected clusters are found then the flow is sent to identify the unselected clusters that follows the further procedure. But, if no unselected cluster is found, then it means that the final cluster head is selected and the flow finally ends. Figure 4 depicts the selected cluster head selected in one of the clusters.

Similarly, the cluster heads can be selected for rest of the clusters. Here, the selected cluster head will have some maximum range of transmission after the selection as shown in the Figure 4. This means that now this cluster head, shown by black dot, can transmit the data to any node in that range of transmission. Thus, by using this methodology, more effective transmission of information can be made possible.

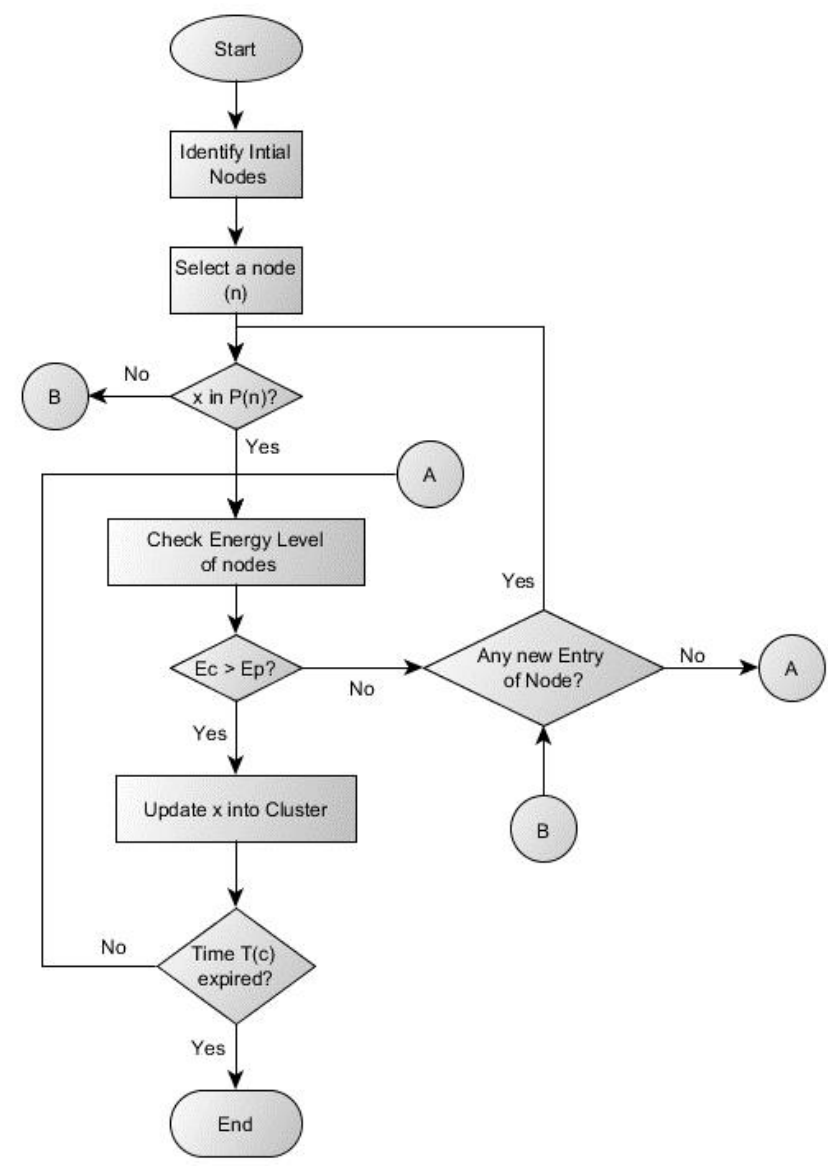

Figure 1. Proposed clustering mechanism

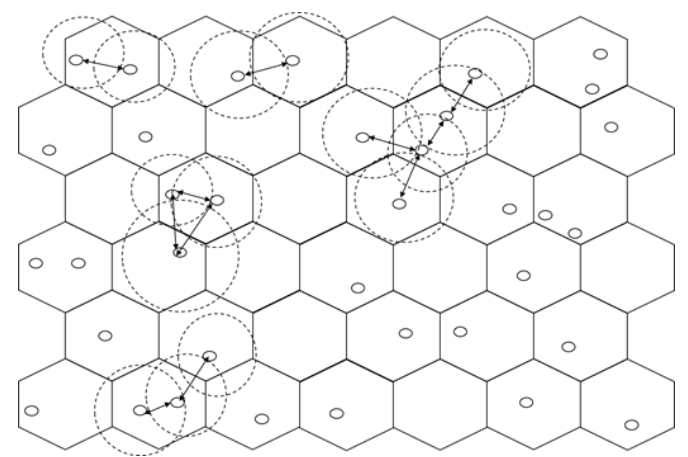

Figure 2. Cluster formation 


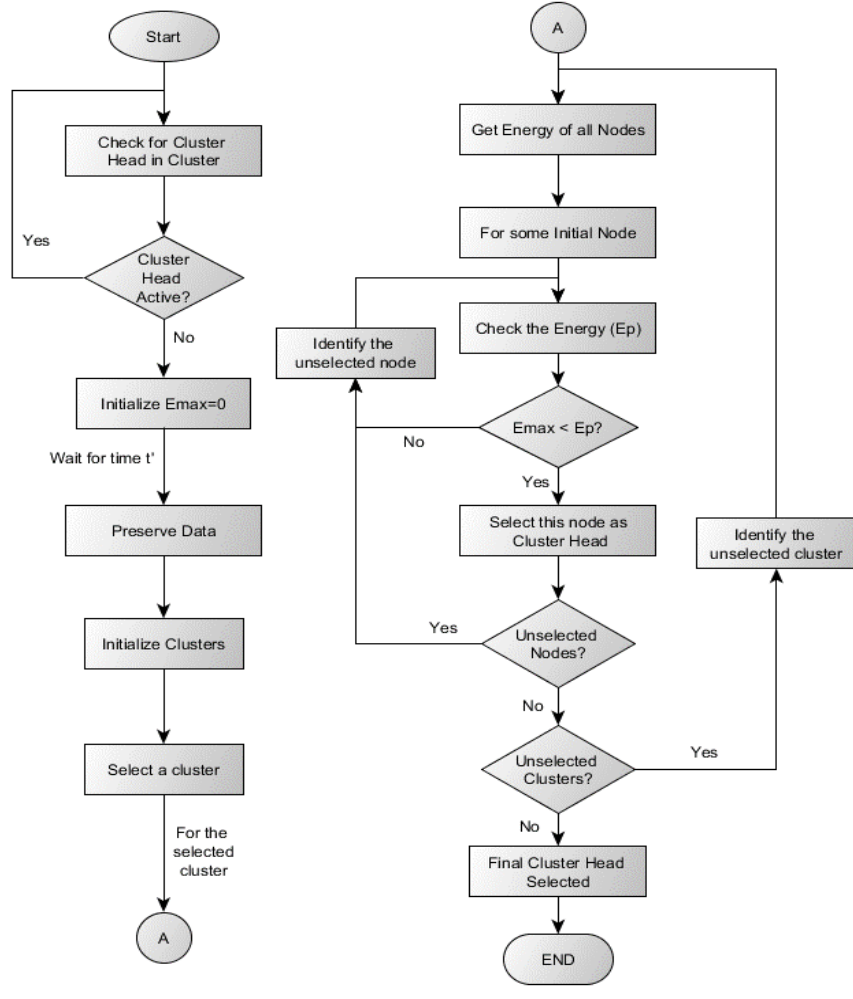

Figure 3. Proposed election mechanism

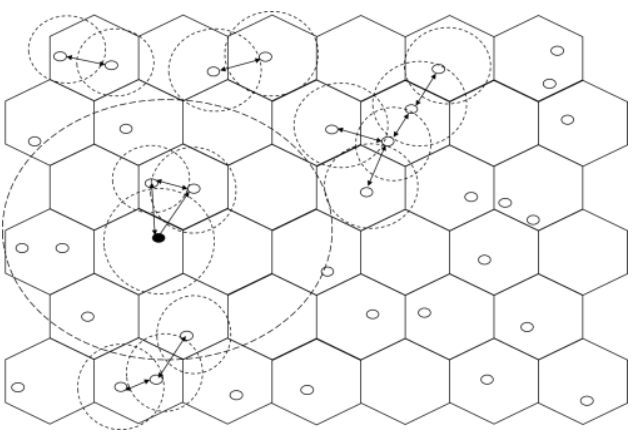

Figure 4. Selected cluster head for a cluster

\section{RESULTS AND ANALYSIS}

In this section, the performance results obtained for the proposed methodology are provided and analyzed with an inference towards the requirement of proposed methodology. The implementation of this research is performed with the help of Network Simulator, NS2 in which a mimic of the actual scenario was designed. Here, the scenario consists of 30 nodes at some position that are moving from source to destination. At some point of time, the cluster formation is initiated and the clusters are formed based on the distance of nodes from each other. Thereafter, the head is elected for each cluster formed. The Intercommunication between various nodes with the help of message communication is shown in the Figure 5(a). After the cluster formation, the packet transfer to the cluster head node is shown in the Figure 5(b).

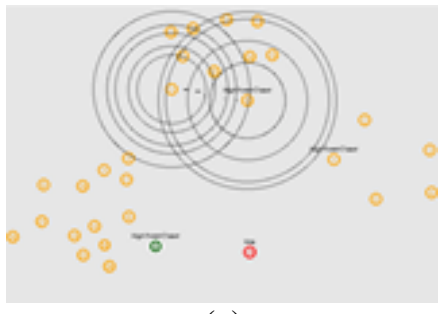

(a)

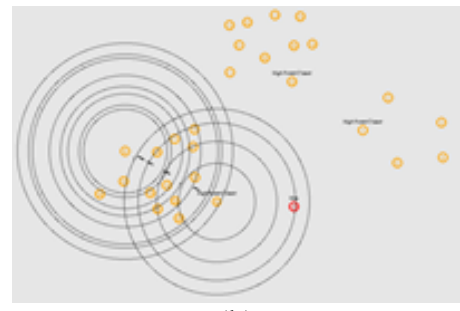

(b)

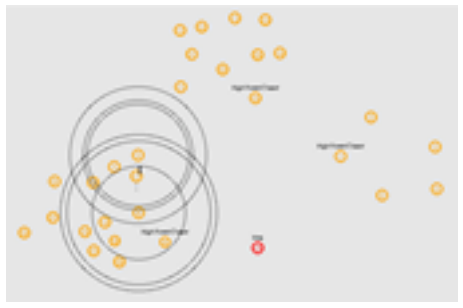

(c)

Figure 5. Simulation using NS2

Now, in terms of packet size vs average throughput of the generating and sending packets as well as the receiving packets, the information is provided in the Table 1 for the cluster head, a node nearer to the cluster head and a node farther from the cluster head. The graph for packet size vs average throughput of sending and receiving at a node nearer to the cluster head are shown in the Figure 6(a) and Figure 6(b) respectively. For the cluster head, Figure 6(c) provides the graph for packet size vs average throughput of receiving packets. With the help of these graphs, the Table 1 is formed that provides the information on the 
average throughput of the sending and receiving packets. It can be analyzed that the average throughput is maximum for the packet size between 0 and 40. Also, it is observed that for the receiving packets of size between 440 and 1000, a linear increase is observed in the average throughput at the cluster head node. Also, for the dropping packets, the average throughput at the cluster head node is observed from the Figure 6.d.

Consider the Figure 5.c that provides the snapshot of a nearer node receiving the data from a node farther to the cluster head and Figure 6.c that provides the packet size vs average throughput of receiving packets at node near to the cluster head. These graphs provide the inference towards the utilization of nodes for transfer of packets from the nodes to the node that is nearer to the cluster head. Comparing all the graphs i.e. for the cluster nodes and the other nodes, the inference towards the receiving of the packets is made by checking to the average throughput of the packets being received at the respective nodes. The maximum value is seen in the case of cluster head followed by node which is nearer to the cluster head. Here, in terms of Simulation End2End Delay, the average delay observed is around 0.165 seconds. Here, for 1 byte, the delay is minimal i.e. 0.000688 , which is negligible. The average number of nodes receiving packets is around 9.09 and the average number of nodes forwarding packets is around 0.0387 .

Table 1. Average Throughput of the Packets

\begin{tabular}{cccc}
\hline Node & Packet & Size (bytes) & Avg. Throughput \\
\hline & Generating & $0-40$ & 15 (Max) \\
& and Sending & $110-120$ & 7 \\
Cluster head & & $0-40$ & 15 (Max) \\
& Receiving & $440-1118$ & Linear Increase \\
& Generating & $0-40$ & 3.5 \\
Node farther & and Sending & $1050-1118$ & Linear Increase \\
from cluster & Receiving & $0-40$ & $\sim 3$ \\
head & & $40-90$ & 1.75 \\
& Generating & $0-40$ & 5 (Max) \\
Node nearer & and Sending & $120-125$ & 0.6 \\
to cluster & & $0-40$ & 4.6 \\
head & Receiving & $40-90$ & 1.9 \\
& & $440-1050$ & Small Linear Increase \\
\hline
\end{tabular}

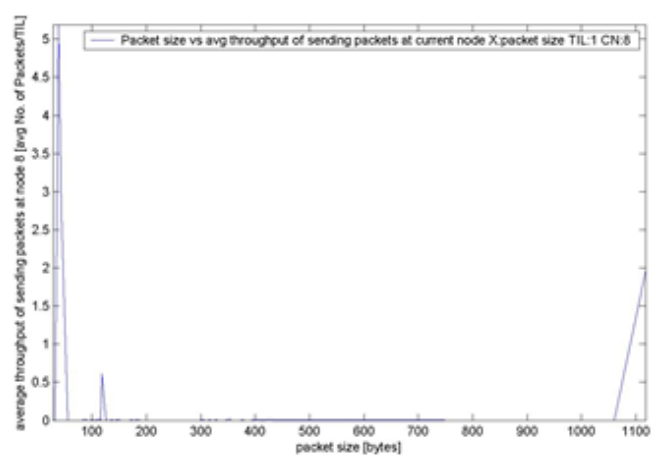

(a)

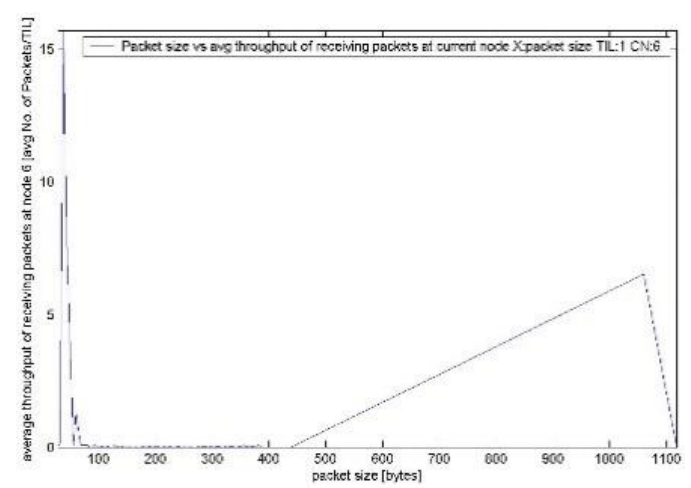

(c)

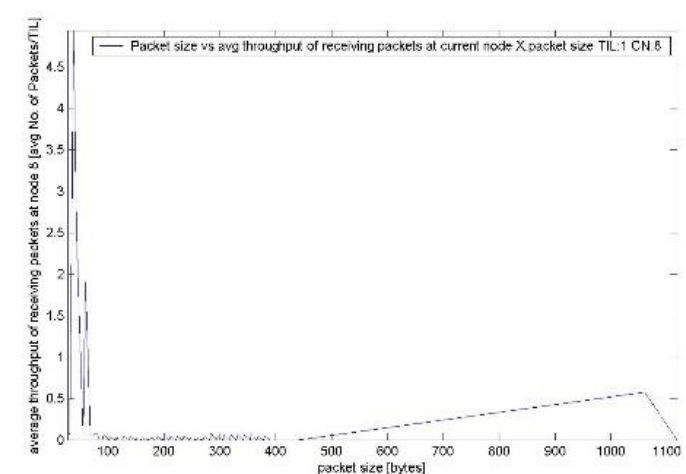

(b)

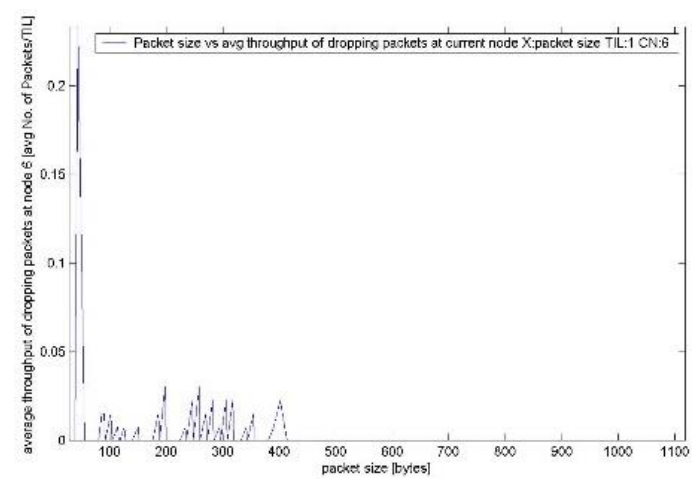

(d)

Figure 6. Simulation results 


\section{DISCUSSION}

The proposed methodology discussed in Section 4 was based on the idea of clustering and bully algorithm. The first objective was to form the clusters considering the distance i.e. radial frequency beacons and the energy of the nodes in the network infrastructures. Then the second objective of the work was to find the cluster head in the formed cluster using the bully algorithm that considers energy and distance as parameters. After these steps, the maximum transfer range of the selected cluster head can be determined for transmission of data considering the dynamic nature of the network nodes. This means that any node can enter into or any node can stop working in an existing cluster at any point of time. In case of any failure based on the above scenarios the election can be performed again using the election algorithm.

Now, consider the points from the literature section. The bandwidth of the network is limited. So, in this methodology, when the bandwidth is getting increased, a greater number of users can be covered. This can also be seen as the introduction of next generation in the cellular networks. Now, considering the point that the number of M2Ds that can be connected are limited, this issue is solved by clustering of the resources being transmitted while keeping the backward compatibility with the original system. But, by doing so, the energy consumption and the delay of access to the GSM may be still not possible for most M2M device. But, in the proposed methodology, as the replication of the data is being performed so, even though the energy will be consumed, the data will be retained.

Finally, from the proposed work and the literature [22-25], it is clear that there is a high need to utilize the dynamic clustering due to mobile nature of the peripheral nodes, so the device will not get out of range even in case the device is moving. The issue of the energy consumption is also solved in this methodology as the data replication will help in retaining the data. As far the infrastructure is concerned, the utilization of the small cells can be helpful in solving the issue of the increasing number of devices. For the discovery of the nodes, the communication between the devices should be initiated. Also, the key point that we are focusing is identification of the nodes by getting the information of the nodes to allow the data to be sent to the other end. Also, this information can also be used for some report analysis and thus it can be seen that with the utilization of cellular network, the object tracking can be performed and as a practical application, with the integration of this approach with IoT modules, it can be applied on roads to ensure follow of rules by tracking down the vehicle which does not follow the rules through multilayer intercommunication provided in this research.

\section{CONCLUSION}

The person who is driving the vehicle abnormally will cause disturbance to the nearest available vehicle, was the reason for the formation of clustering, where the nearest traveling vehicle can be identified. The cluster head that transmit the information regarding the vehicle which serves as the root cause of the abnormality can be send to the next layer/level where things are monitored form the server side. This may easily identify the vehicle and can be removed from the fleet with the help of the past time duration history maintained. The results obtained in this research, provided an inference towards the use of clustering for the object tracking in order to specifically identify the mobile vehicle based on the locality of a node in the cluster. The key inference is towards the interrelation of the vehicles in the learning environment that helps in ensuring a better follow of rules on the road to avoid any mis happening caused due to heavily loaded vehicles or drunken person driving the vehicles.

\section{REFERENCES}

[1] J Dai, J Teng, X Bai, Z Shen and D Xuan, "Mobile Phone Based Drunk Driving Detection", 4th International Conference on Pervasive Computing Technologies for Healthcare, pp. 1-8, March 2010.

[2] W Balzano and F Vitale, "RADS: a smart Road Anomalies Detection System using Vehicle-2-Vehicle network and cluster features", 24th International DMS Conference on Visualization and Visual Languages, July 2018, pp. 1-6.

[3] A Bouchachia, "Dynamic Clustering,", Evolving Systems (2012), Vol. 3, Editorial, pp. 133-134, August 2012.

[4] Abin Paul and Preetha K. G, "A Cluster Based Leader Election Algorithm for MANETs," 2013 International Conference on Control Communication and Computing (ICCC), pp. 496-499, December 2013.

[5] B Ghai, G P Bindalkar, S Sharma, A Shukla, "Energy Efficient Dynamic Nearest Node Election For Localizations of Mobile Node in Wireless Sensor Networks," IEEE International Conference on Computational Intelligence and Computing Research (ICCIC), pp. 1-5, December 2015.

[6] V Kumar, R K Yadav, "Prolonging Network Lifetime by Electing Suitable Cluster Head by Dynamic Weight Adjustment for Weighted Clustering Algorithm in MANET," 3rd International Conference on Computing for Sustainable Global Development (INDIACom), pp. 2915-2920, March 2016.

[7] A Alabass, K Elleithy, A Razaque, "Dynamic Cluster Head Node Election (DCHNE) Model over Wireless Sensor Networks (WSNs)," 29th International Conference on Computers and their Applications, pp. 1-6, March 2014. 
[8] X Cao, H Zhang, Z Shi, G Cui, “Cluster Heads Election Analysis for Multi-hop Wireless Sensor Networks Based on Weighted Graph and Particle Swarm Optimization," 4th Int Conf on Natural Computation, pp. 599-603, 2008.

[9] M A Mehr, "Cluster Head Election Using Imperialist Competitive Algorithm (CHEI) for Wireless Sensor Networks," Int J Mobile Network Communications \& Telematics, Vol. 4, No. 3, pp. 1-9, June 2014.

[10] K Haseeb, K A Bakar, A H Abdullah, "A Review of Cluster Heads Election in WSN," World Applied Sciences Journal Vol. 28 (4), pp. 578-582, 2013.

[11] S. U. Patil, Saste. G. S, Ejagar. G. M, Raskar. S. S, Dhumal P.R, "Dynamic Cluster Head Selection Method using LEACH Algorithm for Wireless Sensor Network," Int J Innovative Research in Electrical, Electronics, Instrumentation and Control Engineering, Vol. 5, Issue 3, pp. 152-154, March 2017.

[12] A Biral, M Centenaro, A Zanella, L Vangelista, M Zorzi, "The challenges of M2M Massive access in wireless cellular networks," Digital Communications and Networks, Volume 1, pp. 1-19, 2015.

[13] C-Y Tu, C.-Y Ho, C-Y Huang, "Energy-efficient algorithms and evaluations for massive access management in cellular based machine to machine communications", IEEE Vehicular Technology Conference, pp. 1-5, 2011.

[14] A. Zanella, N. Bui, A. Castellani, L. Vangelista, and M. Zorzi, "Internet of things for smart cities," Internet of Things Journal, IEEE, Vol. 1, No. 1, pp. 22-32, Feb 2014.

[15] J N Al-Karaki, A E Kamal,"Routing techniques in wireless sensor networks: a survey," Wireless communications, IEEE, Vol. 11, No. 6, pp. 6-28, 2004.

[16] O. Younis and S. Fahmy, "Heed: a hybrid, energy-efficient, distributed clustering approach for ad hoc sensor networks," Mobile Computing, IEEE Transactions on, Vol. 3, No. 4, pp. 366-379, 2004.

[17] L. Qing, Q. Zhu, and M. Wang, "Design of a distributed energy-efficient clustering algorithm for heterogeneous wireless sensor networks," Computer communications, Vol. 29, No. 12, pp. 2230-2237, 2006.

[18] D. Kumar, "Performance analysis of energy efficient clustering protocols for maximising lifetime of wireless sensor networks," Wireless Sensor Systems, IET, vol. 4, no. 1, pp. 9-16, March 2014.

[19] X. Lu, P. Wang, D. Niyato, D. I. Kim, and Z. Han, "Wireless networks with rf energy harvesting: A contemporary survey," Communications Surveys Tutorials, IEEE, Vol. 17, No. 2, pp. 757-789, 2015.

[20] P. Kamalinejad, C. Mahapatra, Z. Sheng, S. Mirabbasi, V. Leung, and Y. L. Guan, "Wireless energy harvesting for the internet of things," Communications Magazine, IEEE, Vol. 53, No. 6, pp. 102-108, June 2015.

[21] Khandakar et al., "Portable System for Monitoring and Controlling Driver Behavior and the Use of a Mobile Phone While Driving," Sensors, MDPI, 19(7): 1563, 2019.

[22] A T Akabane, R Immich, R W Pazzi, E R M Madeira, L A Villas, "Exploiting Vehicular Social Networks and Dynamic Clustering to Enhance Urban Mobility Management," Sensors, MDPI, 19(16), 3558, 2019.

[23] T Han et al., A Hybrid Unequal Clustering Based on Density with Energy Conservation in Wireless Nodes," Sustainability, MDPI, 2019, 11(3), 746.

[24] Bazzi A, Zanella A, "Position based routing in crowd sensing vehicular networks," Ad Hoc Networks, 2016 , Vol. 36, pp. 409-424.

[25] Abuashour A, Kadoch M, "Performance improvement of cluster-based routing protocol in VANET," IEEE Access 2017, 5, pp.15354-15371. 